\title{
EXPERIMENTAL AND NUMERICAL INVESTIGATIONS FOR I-GIRDERS IN BENDING AND SHEAR STIFFENED BY TRAPEZOIDAL STIFFENERS
}

\author{
N. Boissonnade ${ }^{1,{ }^{*}}$, H. Degée ${ }^{1}$, J. Naumes $^{2}$ and M. Oppe ${ }^{2}$ \\ 1ArGEnCo Department, University of Liège, Liège, Belgium \\ ${ }^{2}$ Lehrstuhl für Stahlbau, RWTH Aachen, Aachen, Germany \\ *(Corresponding author: E-mail: N.Boissonnade@ulg.ac.be)
}

Received: 15 January 2007; Revised: 6 April 2007; Accepted: 20 April 2007

\begin{abstract}
The paper investigates the shear resistance of I-girders longitudinally stiffened by trapezoidal stiffeners. It first reports on 4 full-scale tests performed at RWTH Aachen, on stiffened panels with strong closed stiffeners. As expected, failure in the subpanels has been governing. A devoted FEA shell model is then described and validated towards these results and other experimental sources. Parametric studies led with the numerical tool help pointing out several aspects where the Eurocode 3 Part 1.5 recommendations on stiffened panels in bending and shear may be improved.
\end{abstract}

Keywords: Stiffened plate, trapezoidal stiffener, experimental test, numerical model, shear buckling

\section{INTRODUCTION}

This paper presents part of the research work developed in the frame of a European RFCS project designated as "ComBri" [1] (Competitive Steel and Composite Bridges by improved Steel Plated Structures). Within this project, several aspects of steel and composite bridge design such as resistance to bending, to shear, to patch loading or bridge launching have been studied.

Present paper focuses on the problem of stiffened web panels in shear or in combined bending and shear, the use of strong closed longitudinal stiffeners becoming more and more widespread since it is shown to allow for a more economic design. The main purpose is here to improve the design of longitudinally stiffened I-girders so that to benefit from the increased resistance of panels stiffened by a few strong closed stiffeners. Indeed, web subpanels adjacent to a closed (trapezoidal) stiffener may be significantly strengthened by the high torsional stiffness of the longitudinal stiffener that improves the subpanels' resistance to shear buckling. In addition, buckling of the whole stiffened panel may be significantly restrained by the use of a trapezoidal stiffener. The problem is here to determine in what extent this may positively affect the resistance of the whole girder.

In this field, Eurocode 3 Part 1.5 [2] appears to be one of the most advanced set of design rules. It proposes general recommendations, but the particular case of closed stiffeners is not especially addressed. Part 1.5 rules are indeed based on the so-called "rotated stress field method", that have first been developed for unstiffened webs with large aspect ratios. Then, the method has been further extended to longitudinally stiffened girders, and validated through tests and FEA numerical calculations. Nevertheless, it seems that very few developments and data towards the derivation of specific rules for strong closed stiffeners have been collected.

In this respect, this paper first briefly reports on a series of four shear buckling tests performed at RWTH Aachen, where the test specimens were designed with strong longitudinal trapezoidal stiffeners ensuring a local failure in the subpanels. Then, the paper describes the development and assessment of FEA shell models towards several sources of experimental data. On the basis of the developed numerical tools, parametric studies have been conducted, and the results of a comparison between these numerical results and the application of Eurocode 3 Part 1.5 rules are presented. 
Conclusions on the ability of the code to provide safe and accurate results when the girder comprises longitudinal closed stiffeners are finally given.

\section{SHEAR BUCKLING TESTS (RWTH AACHEN)}

Four full-scale tests on I-girders with slender webs have been performed at RWTH Aachen [3]. They consist of 3-point bending tests (see Figure 1) prepared and performed to serve as a reference for the validation of FEA models. They have been designed so that to exhibit the increase of resistance brought by a strong closed longitudinal stiffener. Accordingly, two specimens were designed with non-stiffened webs while the other two have been prepared with the same material and dimensions but also with a longitudinal stiffener.

The longitudinal stiffeners have been chosen so that to lead to a low global panel slenderness (i.e. including the restraining effect of the stiffener) that ensures failure in the subpanels. Therefore, the beneficial effect of the stiffener is maximised [1].

The material properties of the tested specimen are given in Table 1.

Table 1. Material Properties

\begin{tabular}{|c|c|c|c|c|}
\cline { 2 - 5 } \multicolumn{1}{c|}{} & Web & Flanges & Longitudinal stiffener & Transverse stiffener \\
\hline$E[\mathrm{GPa}]$ & 210 & 210 & 210 & 210 \\
\hline$f_{y}[\mathrm{MPa}]$ & 399 & 433 & 399 & 433 \\
\hline
\end{tabular}

Different measurement devices have been used during the tests. They have been chosen so that to get exploitable information for further comparison with numerical simulations. In addition to the load cell, 6 strain gauges and 10 3D-rosettes have been placed on the specimens (see Figure 2). Furthermore, photogrammetric measurements by means of three digital cameras have been used to determine the spatial evolution of displacements during loading. For that purpose, a regular grid of targets has been placed on the web surface (Figure 2). The three-dimensional displacements have been obtained through an appropriate numerical treatment of two or three images. This photogrammetric system has also been used to provide information on the initial geometrical imperfections.

Figure 3 reports on the obtained load-displacement curves. As can be seen, the results obtained with the stiffened girders (Test $2 \mathrm{a}$ and Test $2 \mathrm{~b}$ ) are significantly higher than with the unstiffened girders (Test 1a and Test $1 \mathrm{~b}$ ). This clearly points out how much additional resistance can be provided through the use of a longitudinal stiffener being able to prevent global panel buckling. These results are further compared with FEA results in the next section. 


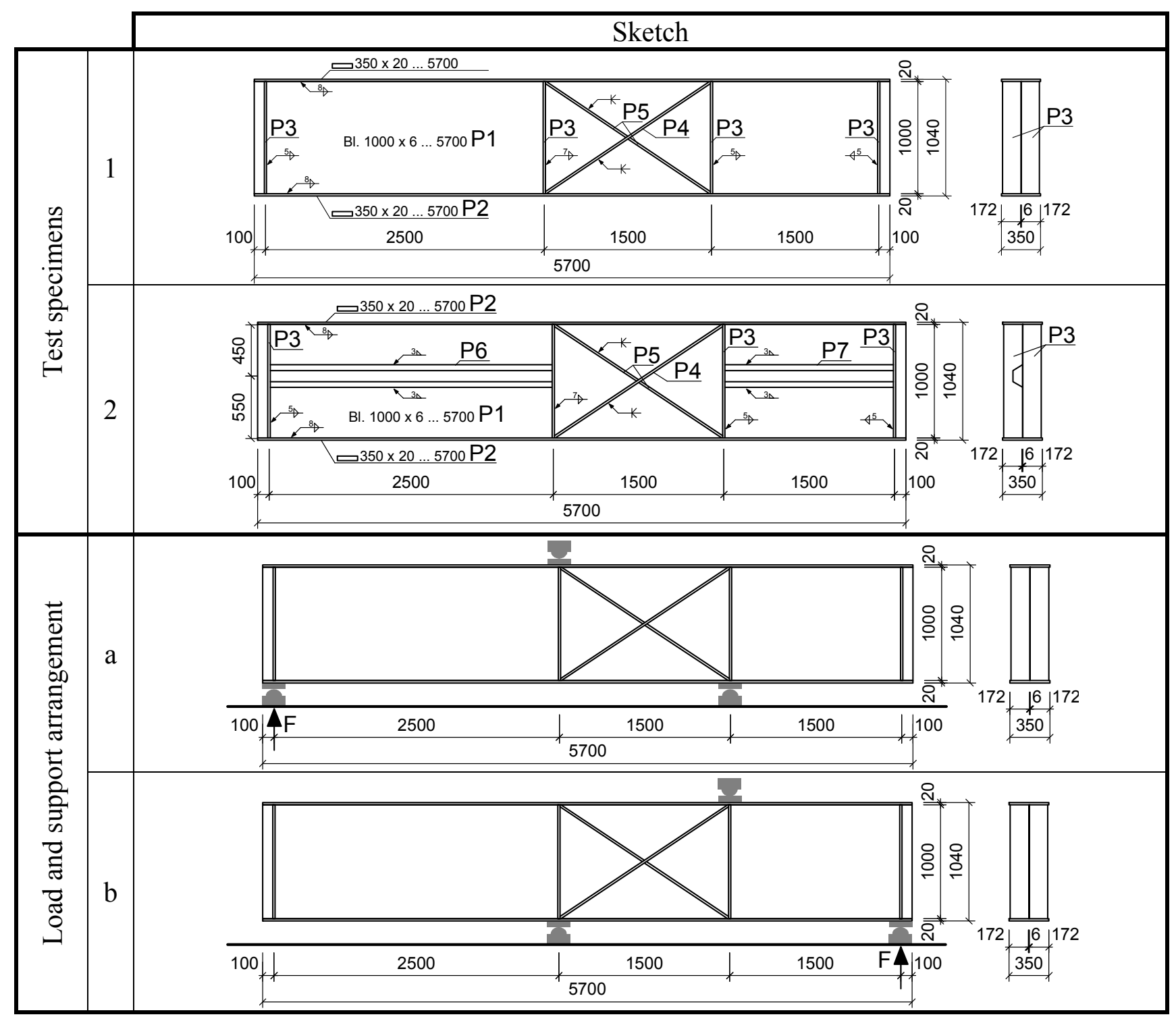

Figure 1. Test Program for Shear Buckling Tests
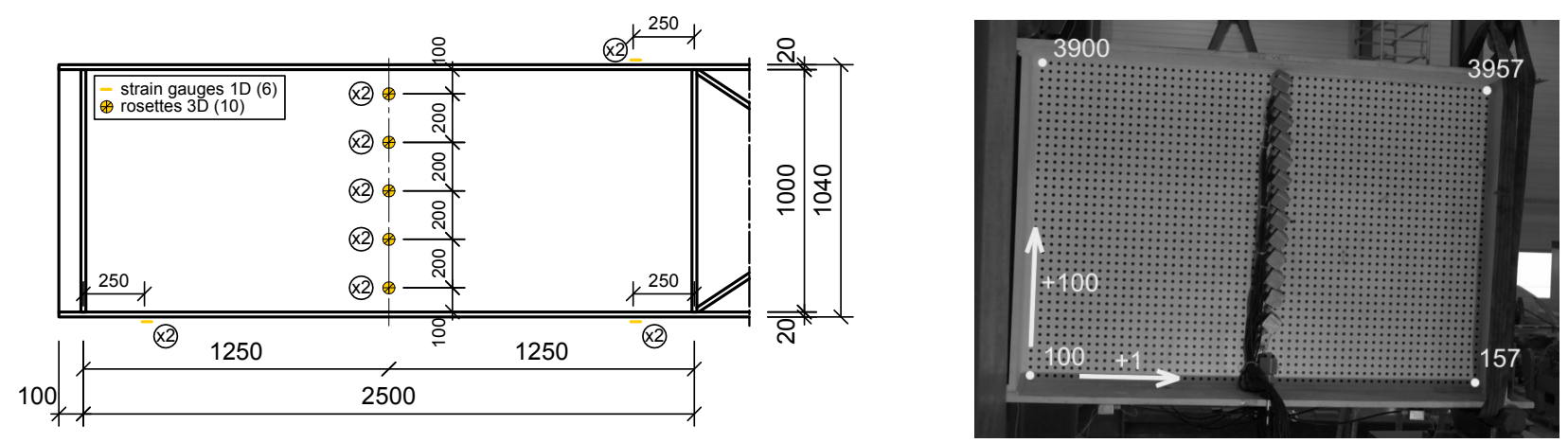

Figure 2. Rosettes and Strain Gauges Locations - Test Grid of Targets 


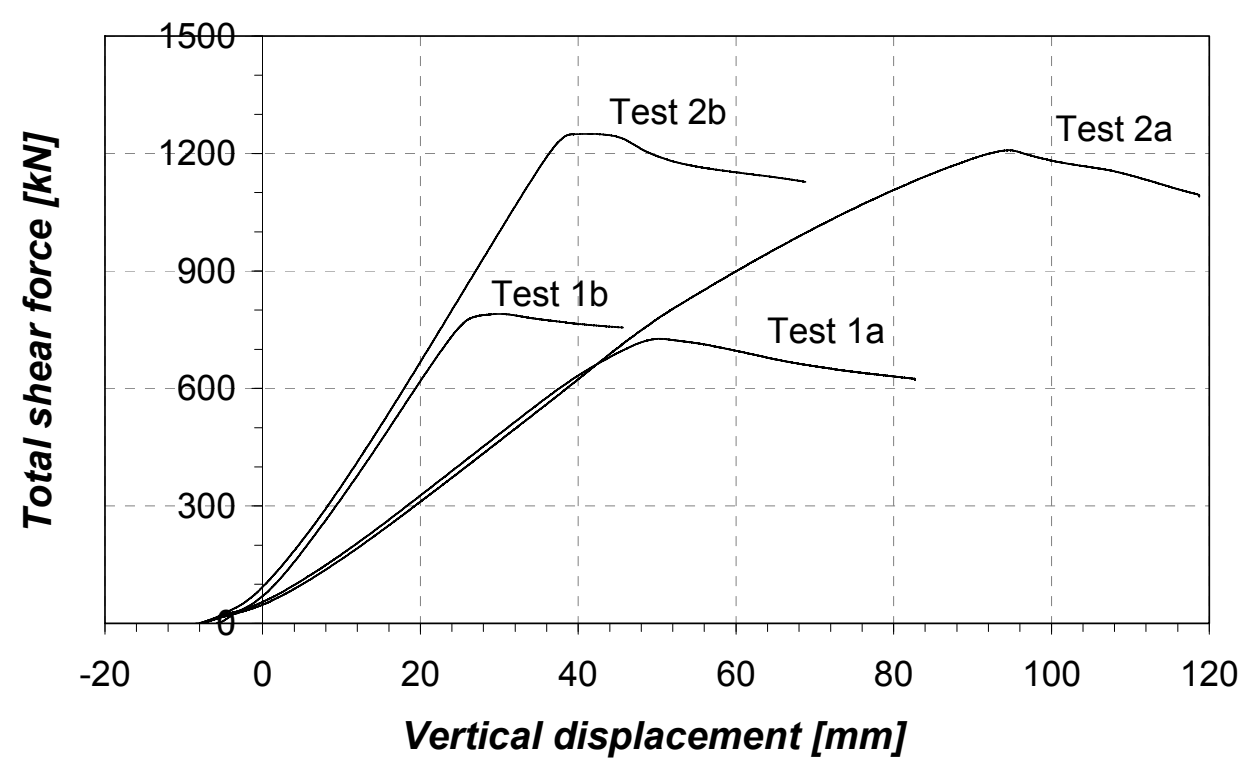

Figure 3. Experimental Load-displacement Curves

\section{DEVELOPMENT AND ASSESSMENT OF FEA MODELS}

\subsection{Description of the FEA Models}

Several FEA non-linear models have been derived both at RWTH Aachen with MARC-Software and at M\&S Liège with FINELg. Because the uses of both software lead to very close results, no distinction is made here from the different numerical sources.

The geometry of the I-girder is accounted for by means of shell elements, with mesh densities that have been shown to be sufficient for a reasonably accurate calculation of a shear buckling situation (see Figure 4). The support conditions are derived in order to fulfil the three-point bending load case, and the applied force consists in a linearly distributed load along the flange width, as was done in the experimental tests. The material is assumed as elastic-plastic with no strain hardening, and residual stresses are not accounted for. Deeper details on the shell elements and modelling assumptions may be found in [4].
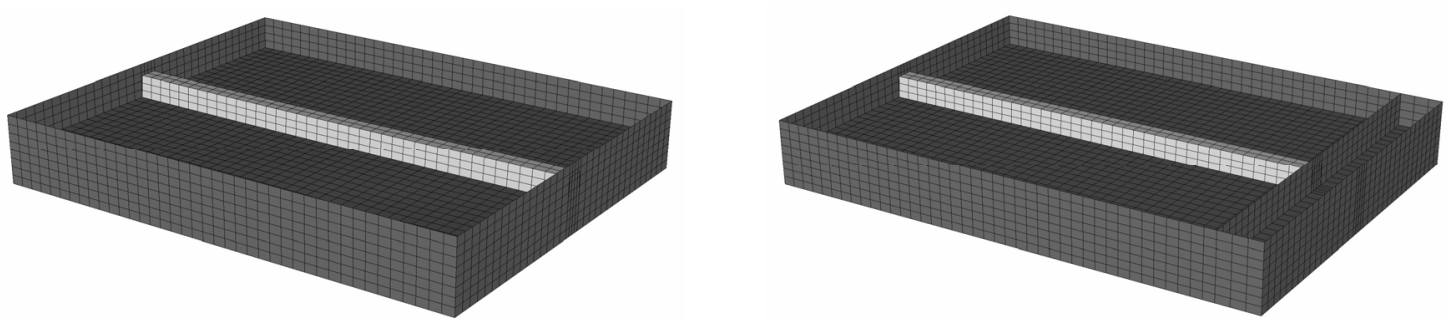

Figure 4. FEA Shell Models: Simple Model (RWTH Tests) and Refined Model (Stuttgart Tests)

As can be seen on Figure 4, two different FEA models have been derived: a first simple model that is dedicated to the simulation of the RWTH Aachen tests, and a more refined model allowing dealing with rigid or non-rigid end-posts.

As a particular point, the introduction of geometrical imperfections in the FEA calculations is worthy of further discussion. Indeed, several possibilities to combine global panel default with local subpanels imperfections were seen to lead to non-negligible discrepancies on the carrying capacity of the girder. 
In accordance with Eurocode 3 Part 1.5 recommendations for FEA modelling, both global and local imperfections have been taken into account. The global imperfection was assumed to be a bow imperfection with maximum amplitude equal to $a / 400, a$ being the length of the web panel. The local subpanel imperfection has to be set affine to the considered subpanel buckling shape, with an amplitude of $\min (a / 200 ; b / 200)$, where $a$ and $b$ are the dimensions of the subpanel.

Then, several combinations of these isolated defaults may be defined. According to Eurocode 3, a leading imperfection has to be accompanied by a secondary imperfection with $70 \%$ of its recommended amplitude. The determination of the imperfection that should be considered as the major one then implies to perform two calculations where the global and local defaults play the major role alternatively. In addition, the direction of each imperfection has to be defined since each one can be either on the same side as the stiffener or on the opposite side. As a consequence, several calculations need to be achieved, and the minimum obtained capacity shall be kept as the reference value.

It has been shown [1] that, concerning the present problem, the combination where the global subpanel imperfection is chosen as the major imperfection on the stiffener's side together with a local default on the opposite side lead to the most conservative results.

Besides this "code" combination procedure, a second method to account for the geometrical imperfection has been used. It consists of using the displacements pattern from the last step of the "code" non-linear calculation (i.e. in the post-buckling range) with adequate amplitude as the initial imperfection. This allows studying directly the "worst" imperfection shape where global and local imperfections are merged. An amplitude equal to $a / 800$ has been shown to be appropriate and safe, the influence of the shape being more important than the value of the initial amplitude.

The influence of initial residual stresses has not been taken into account, since it is known to have a very small influence on this type of results, especially regarding shear behaviour where the welding affects a quite limited zone [1].

\subsection{Assessment of FEA Models}

The results obtained from the numerical model have been compared with the experimental results. Figure 5 presents the obtained load-displacement curves, where the FEA calculations have been achieved through the "code" procedure described in Section 3.1.

As can be seen, the different FEA curves are in quite good agreement with their respective experimental counterparts. It is to be noted that a good correspondence for the whole load-displacements curves has only be made possible through an appropriate modelling of the supports with non fully-rigid but elastic supports, the spring stiffness of the latter being the measured ones.

Finally, additional numerical calculations have been performed with the "real" initial geometrical imperfection pattern, see Figure 6. Indeed, as explained in Section 2, the photogrammetric system has been prepared so that to be able to provide such data. A linear interpolation between the measurements got from the physical test grid of targets and the FEA model mesh has been used to convert the necessary data in the numerical model. Figure 7 compares the results of these calculations with those obtained in the "code" procedure. 


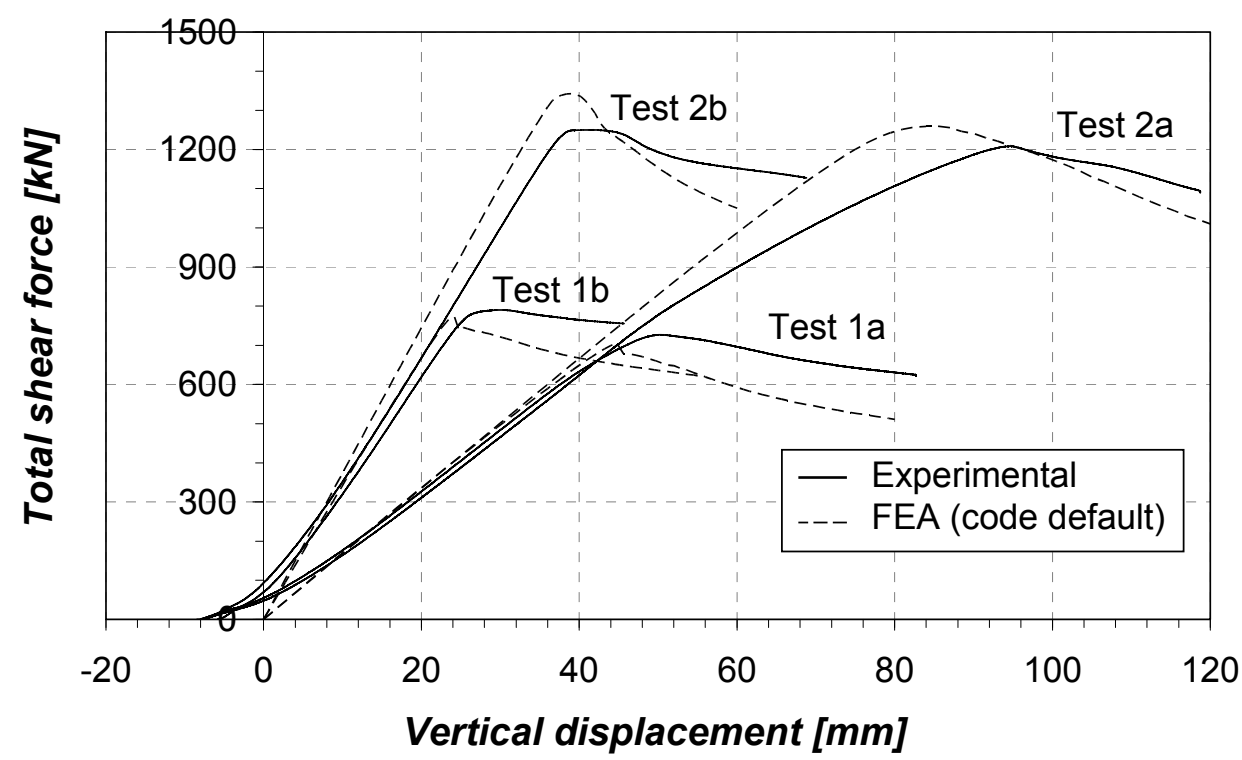

Figure 5. FEA vs. Test Load-displacement Curves
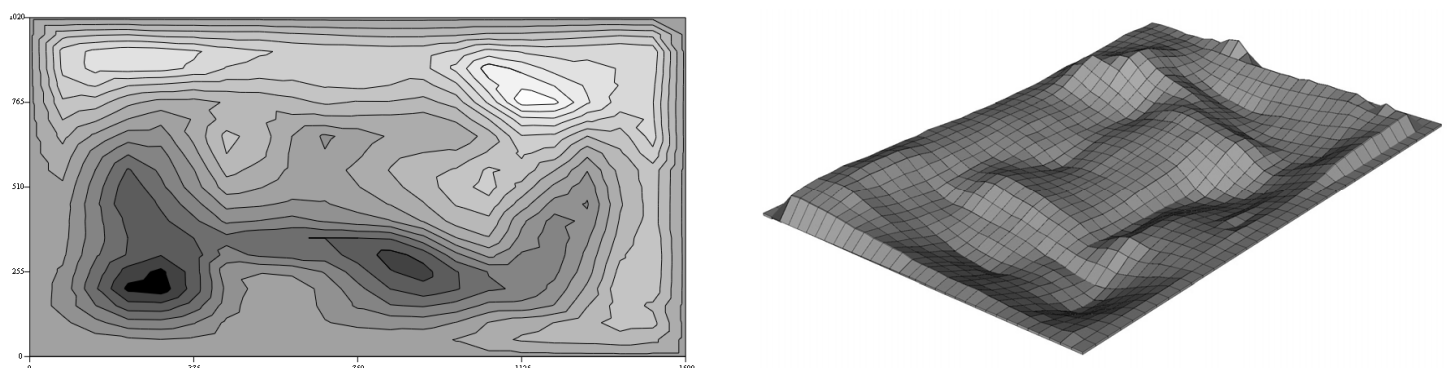

Figure 6. Measured Distribution of Initial Imperfections and Associated FEA Mesh (Amplified)

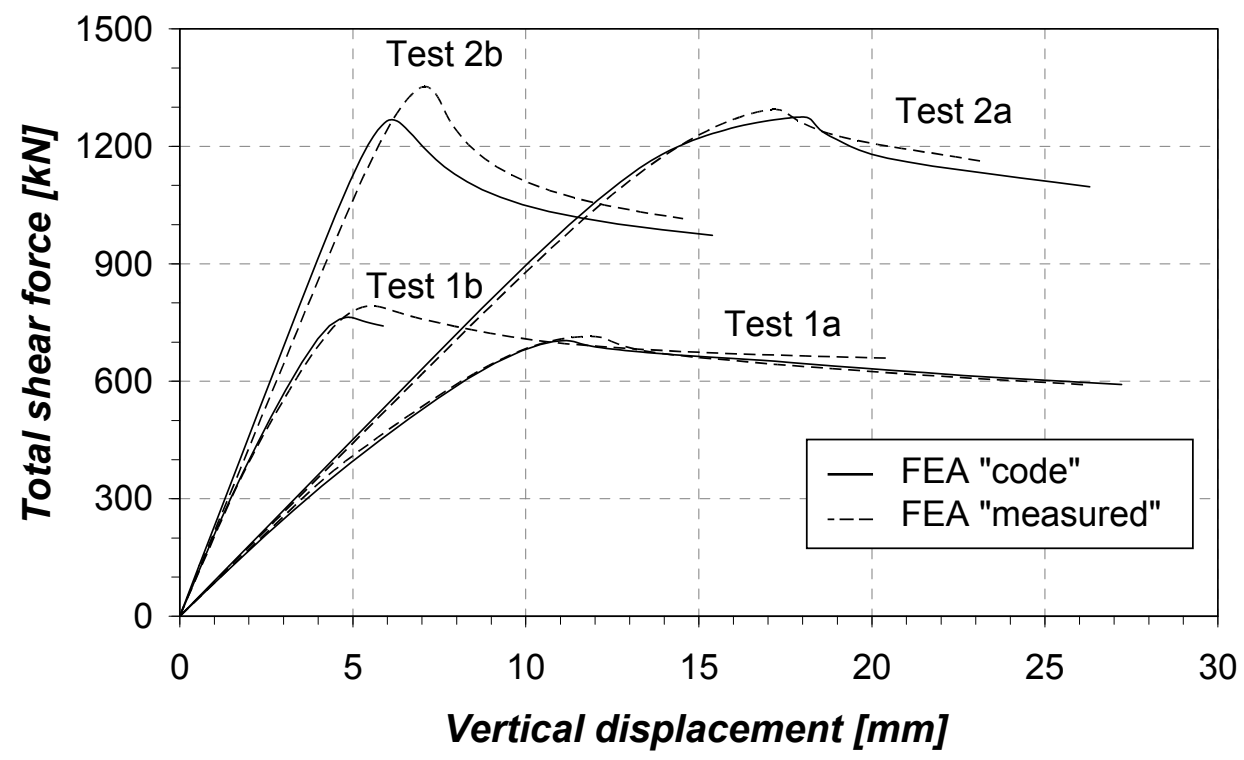

Figure 7. FEA Results for "Code" and Measured Imperfections (No Springs at Supports)

As a very good accordance between all calculations is observed, the different procedures described above to deal with the geometrical imperfections are found appropriate. 
In order to further assess the ability of the models to simulate accurately the shear buckling behaviour of I-girders, additional comparisons have been carried out with other similar tests performed in Stuttgart ([5], [6], [7]).

In these four "Stuttgart tests", the longitudinal (closed) stiffeners dimensions have been chosen so that to cover different situations, from weak to strong stiffeners. So-called "weak" stiffeners are such that they buckle together with the subpanels when shear buckling occurs (i.e. global panel buckling), while stronger stiffeners restricts shear buckling to the weakest subpanel.

Table 2. Results for Stuttgart Shear Tests (Loads in $\mathrm{kN}$ )

\begin{tabular}{|c|c|c|c|c|}
\hline Girder & $G_{1}$ & $G_{2}$ & $G_{3}$ & $G_{4}$ \\
\hline Experimental & 1453 & 1569 & 1412 & 1591 \\
\hline FEA code & 1494 & 1524 & 1428 & 1468 \\
\hline FEA post & 1408 & 1446 & 1355 & 1408 \\
\hline$\%_{\text {FEA code }}$ & -2.7 & 3.0 & -1.1 & 8.4 \\
\hline \%FEA post & 3.2 & 8.5 & 4.2 & 13.0 \\
\hline
\end{tabular}

As can be seen in Table 2, the correspondence between experimental and numerical results is quite good. The design of these tests being completely different from the tests performed in Aachen, the quality of the obtained results further ensures the reliability of the developed FEA models. On the basis of these results, the models are found safe and accurate. They have been extensively used in the parametric studies described in the following.

\section{PARAMETRIC STUDIES}

The validity of the FEA models being confirmed, parametric studies have been led with respect to a 3-point bending arrangement represented in Figure 8. They are mainly dedicated to the shear behaviour of the stiffened girder.
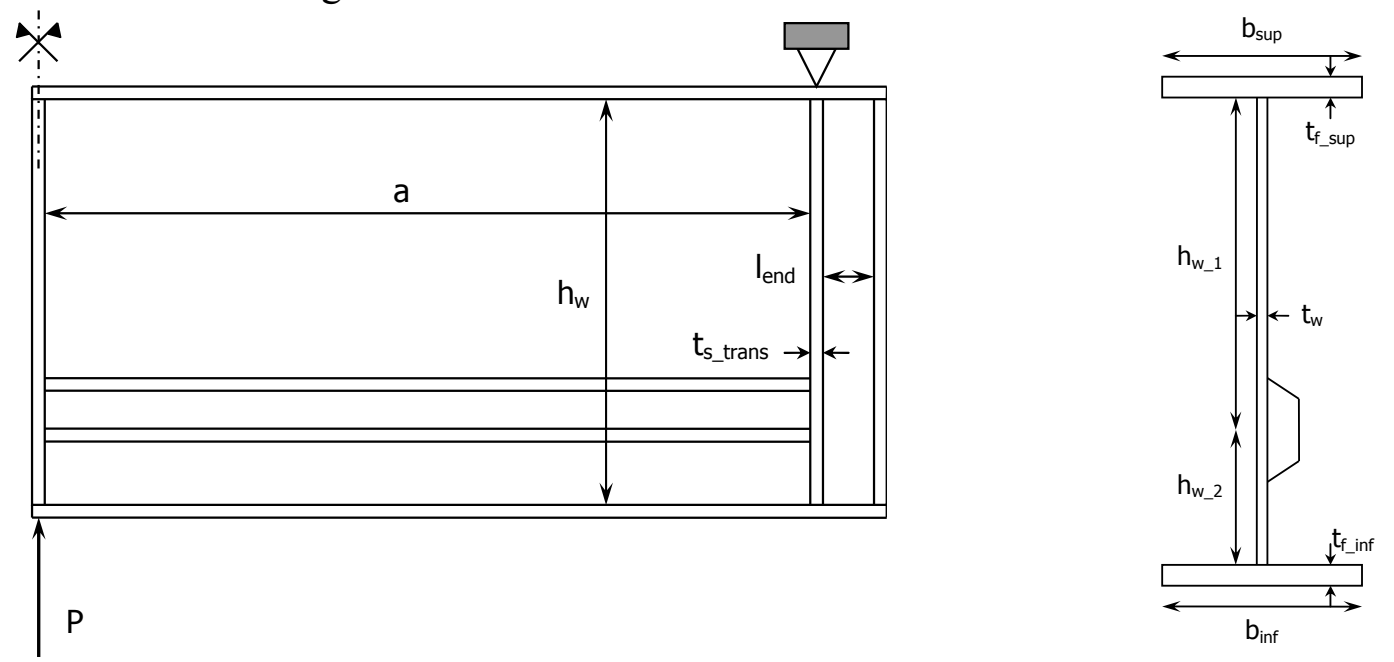

Figure 8. Loading, Support Conditions and Geometrical Definitions for the Investigated System

The following parameters have been taken into account in a first parametric study:

- 2 different trapezoidal stiffeners ("weak" and "strong");

- Web thickness equal to 6 or $12 \mathrm{~mm}$;

- 3 aspect ratios for the web: 1.0, 3.0 and 5.0;

- 2 web depths $h_{w}: 1000$ and $2000 \mathrm{~mm}$; 
- 2 positions for the longitudinal stiffener: at $0.5 h_{w}$ or $0.3 h_{w}$;

- Rigid or non-rigid end-posts;

- "Code" or "post" initial geometrical imperfection.

- Closed or open trapezoidal stiffeners, or no stiffener;

In addition, a second parametric study on stiffened girders with higher web depths has been led, according to the following parameters:

- 2 different trapezoidal stiffeners ("weak" and "strong");

- Web thickness equal to $20 \mathrm{~mm}$;

- 3 aspect ratios for the web: $1.0,1.5$ and 2.0

- 3 web depths $h_{w}: 2000,3000$ and $4000 \mathrm{~mm}$;

- 2 positions for the longitudinal stiffener: at $0.5 h_{w}$ or $0.3 h_{w}$;

- Rigid or non-rigid end-posts;

- "Code" or "post" initial geometrical imperfection.

- Closed or open trapezoidal stiffeners, or no stiffener;

As both "code"- or "post"-type initial imperfections calculations have been performed, the lowest ultimate loads have been kept as the FEA reference. In total, 1260 FEA-shell non-linear calculations have been performed (refined model).

Cases where the trapezoidal stiffener is said to be "open" aims at testing the influence of the torsional stiffness brought by the longitudinal stiffener. For such particular cases, the flange of the trapezoidal stiffener is assumed to be cut in its middle; as a consequence, the stiffener, still of trapezoidal shape, only brings a torsional stiffness corresponding to that of an open section, i.e. that is much lower than for a closed section shape.

All the results provided by the parametric studies have been compared to the corresponding Eurocode 3 Part 1.5 results. According to the code, the following resistance checks have to be performed within the present loading and support conditions:

- Bending resistance check at mid-span with a gross (Class 3) cross-section ("Gross");

- Bending resistance check at a distance equal to min $(0.4 a ; 0.5 b)$ from mid-span ("Bending");

- Shear buckling resistance check ("Shear");

- Possible interaction between bending and shear at a distance equal to $0.5 h_{w}$ from mid-span ("Interaction").

All Eurocode 3 Part 1.5 results presented in the following correspond to the minimum load obtained in accordance with the four above-listed criteria.

Besides a strict application of Eurocode 3 rules, additional code-type calculations have been performed, where the buckling stresses are determined numerically. Eurocode 3 Part 1.5 indeed offers the opportunity to calculate separately the elastic plate buckling stresses, and use of EBPlate software has been made [8]. It allows getting quite accurate numerical values in many situations, and, in particular, dealing with the influence of the longitudinal stiffeners on global panel behaviour [9] or the beneficial effect of flanges and transverse stiffeners.

Accordingly, three different code-type calculations are presented in the following Figures:

- The first one corresponds to a strict application of Eurocode 3 recommendations, i.e. in line with the procedures proposed in Part 1.5 Annex A for the evaluation of bending and shear critical stresses ("Pure EC3"); 
- A second calculation includes the beneficial effect of the girder's flanges on the stability of the adjacent panels (including possible shear lag in the flanges), that may no more be considered as simply supported ("EC3 2 edges");

- A third calculation takes in addition advantage of the transverse stiffeners on the global panel stability ("EC3 4 edges").
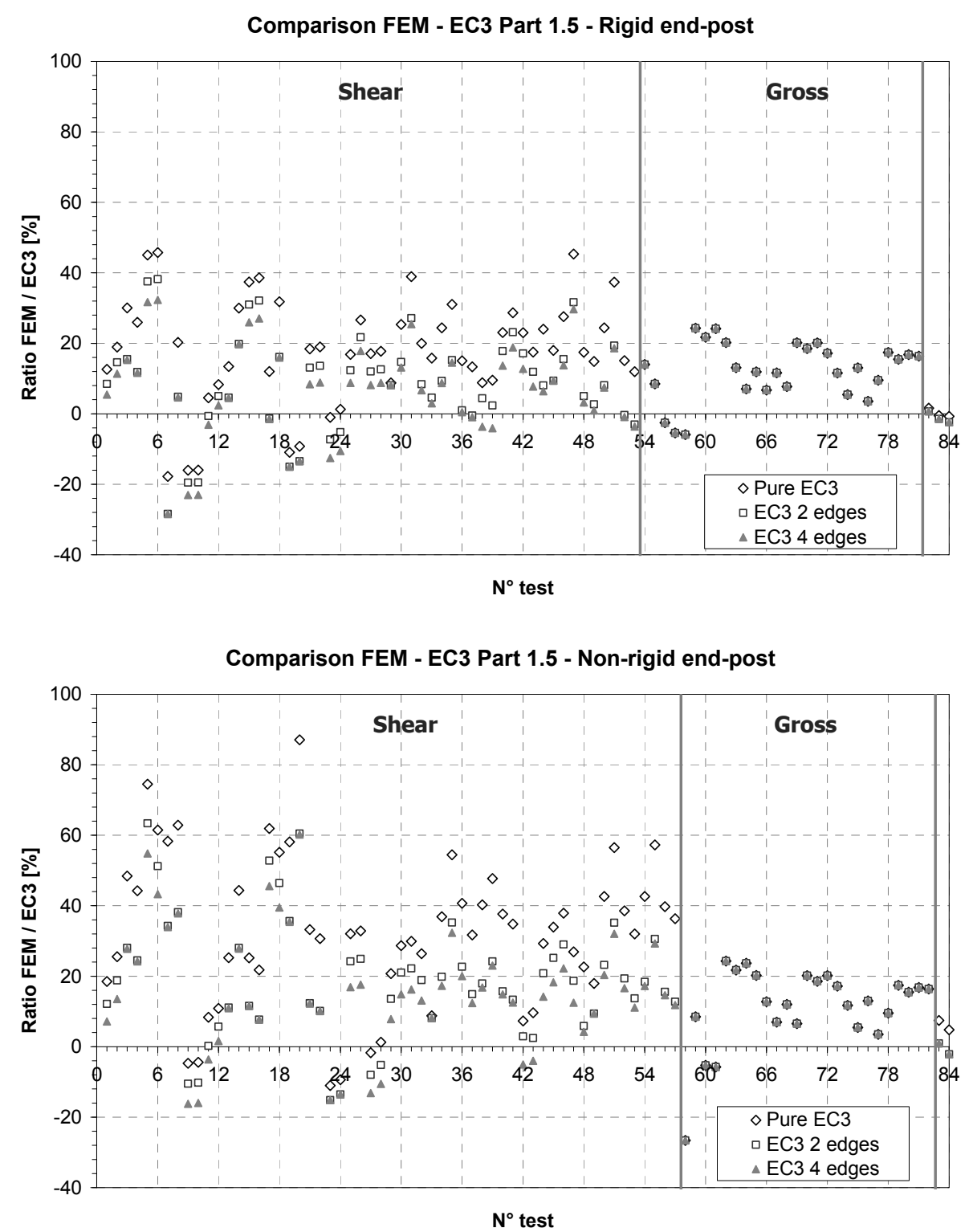

Figure 9. Comparison of Results for Closed Stiffeners

Figure 9 reports on the accuracy of these three approaches for respectively rigid and non-rigid end-post cases, when the results are sorted by failure type (according to Eurocode 3 criteria). On this Figure, the different geometric situations tested are plotted versus the percentage of difference in ultimate loads between the code result and the reference FEA result.

It is first seen that the "Shear" and "Gross" failure modes mainly govern in the different situations chosen. Then, it clearly appears that the results provided in the rigid end-post situation are much more accurate than in the non-rigid end-post case, in comparison with the FEA references. Whereas the levels of accuracy and safety appear to be quite acceptable when the "Gross" criterion governs (i.e. girders with high aspect ratios $\alpha$ ), situations where shear is the leading phenomenon exhibit a highly varying level of accuracy, even more for the non-rigid end-post cases. That may be explained 
by the fact that the trapezoidal shape of the stiffener significantly improves the lateral resistance of the transversal stiffener of the non-rigid end-post. Therefore, no penalty on the shear buckling resistance of the stiffened web should be applied, as is nevertheless recommended by Eurocode 3 Part 1.5 rules.

It is also shown, as expected, that when the calculations of critical stresses get more and more refined, the obtained results get more and more accurate. Figure 10 also leads to the same conclusion in case of an open stiffener. In cases where the web panel is unstiffened, the distinction between rigid and no-rigid end-post appears to be justified [1].
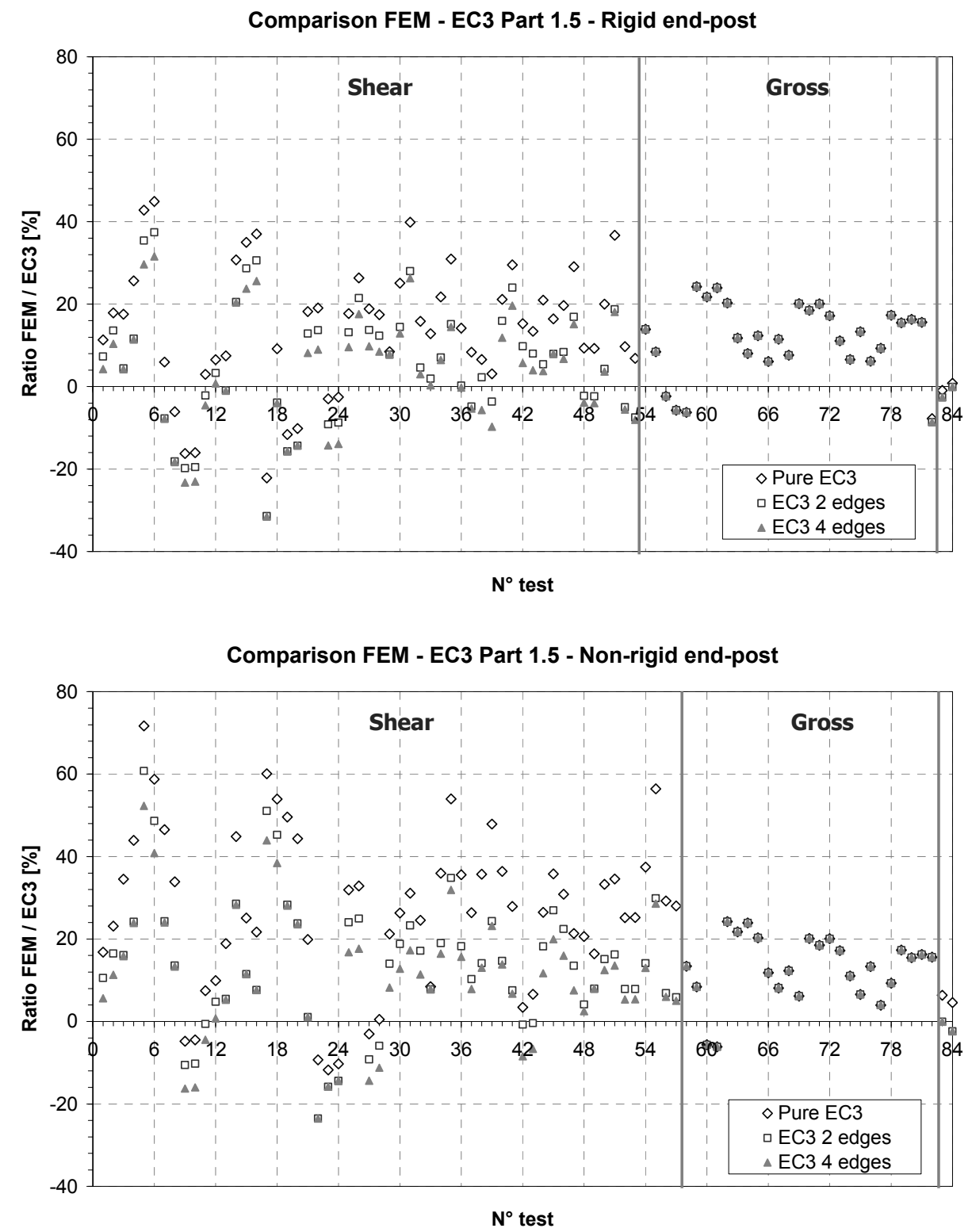

Figure 10. Comparison of Results for Open Stiffeners

Figure 11 proposes another plotting of the results, as a function of the global web relative slenderness to shear $\lambda_{w}$. This permits to exhibit in which extent an accurate determination of the critical stress positively affects the value of $\lambda_{w}$, thus the carrying capacity. It appears however that Eurocode 3 Part 1.5 rules may lead to slightly unsafe results for low value of $\lambda_{w}$. This was shown to be due to the fact that Part 1.5 accounts for a beneficial effect of strain hardening on the shear resistance that was disregarded in the FEA calculations. 

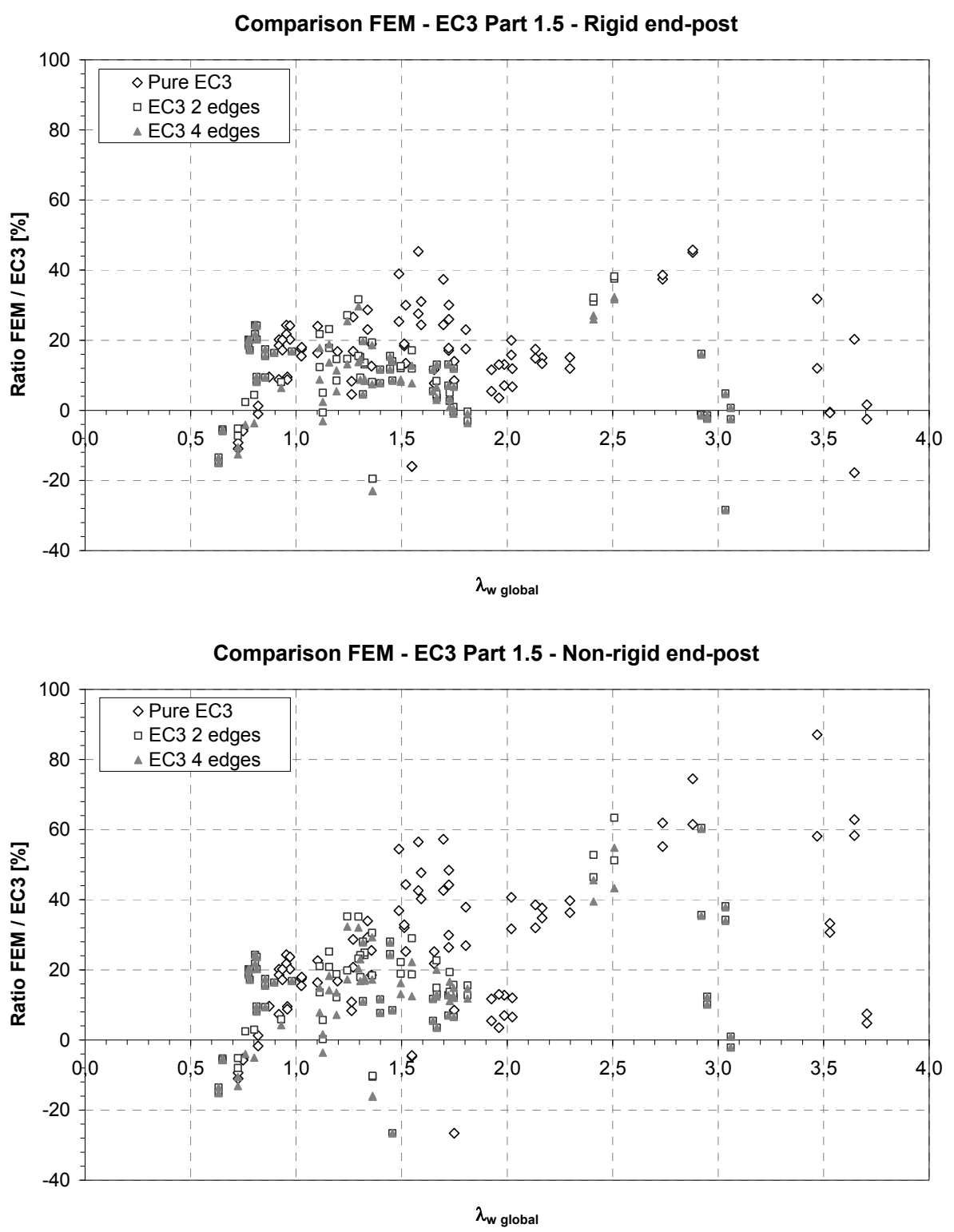

Figure 11. Influence of the Determination Procedure for Critical Stresses (Closed Stiffener)

\section{CONCLUSION}

Through extensive results from a validated numerical FEA tool, the paper has shown that the Eurocode 3 Part 1.5 recommendations could be improved for the specific cases of I-girders in shear stiffened by closed trapezoidal stiffeners. Indeed, especially for non-rigid end-post situations, the results provided by the application of Eurocode 3 Part 1.5 rules exhibit over-conservative design that could be improved. This may be due to the quite stiff shape of the trapezoidal stiffener since results for open stiffener also lead to the same conclusion.

In addition, it is shown that the possibility offered by the code to calculate separately (i.e. from numerical sources) the critical plate buckling stresses lead to more satisfactory results, for all cases involving longitudinal stiffeners.

Several possibilities for improvement in this way are actually under development, and are planned to be achieved within the end of the "ComBri" project. 


\section{ACKNOWLEDGEMENTS}

The authors deeply acknowledge the Research Fund for Coal and Steel (RFCS) of the European Union for their financial support.

\section{REFERENCES}

[1] ComBri 2004 - 2006, RFCS Contract n RFS-CR-03018 “ComBri”, Technical Reports n 4-5-6, 2004-2006.

[2] Eurocode 3 Part 1.5 2004, prEN1993-1-5: Design of Steel Structures - Plated Structural Elements, 2004.

[3] Naumes, J., Oppe, M., "ComBri" Competitive Steel and Composite Bridges by Improved Steel Plated Structures. Test Report - Shear Buckling Tests. RWTH Aachen, 2006.

[4] Boissonnade, N., Degée, H., "ComBri" Competitive Steel and Composite Bridges by Improved Steel Plated Structures. Background Document to Work Package 1.2 - Numerical and Code Aspects, University of Liège, 2006.

[5] Pavlovcic L., Seitz M., Detzel A., Kuhlmann U., Beg D., "Report on Tests and FE Study on Longitudinally Stiffened Web of Plate Girders in Shear", Institut für Konstruktion und Entwurf, Stahl- Holz- und Verbundbau, Universität Stuttgart, 2003.

[6] Pavlovcic L., Detzel A., Kuhlmann U., Beg D., "Shear Resistance of Longitudinally Stiffened Panels - Part 1: Tests and Numerical Analysis of Imperfections, Journal of Constructional Steel Research, March 2007, Vol. 63, Issue 3, pp. 337-350.

[7] Pavlovcic L., Detzel A., Kuhlmann U., Beg D., "Shear Resistance of Longitudinally Stiffened Panels - Part 2: Numerical Parametric Study", Journal of Constructional Steel Research, March 2007, Vol. 63, Issue 3, pp. 351-364.

[8] EBPlate, "Elastic Buckling of Plates", Software Developed by CTICM in the Frame of the ComBri Project, RFCS Contract n RFS-CR-03018, 2006.

[9] Galéa Y., Martin P.O., "Plate Type Behaviour - Use of EBPlate to Calculate $\sigma_{\text {cr,p. }}$. Working Document", Stuttgart Meeting, December 2005. 